\title{
Seroprevalence of cytomegalovirus among pregnant women in Windhoek, Namibia, 2016
}

\author{
B E van der Colf,, ${ }^{1,2}$ MSc; G U van Zyl, ${ }^{2,3}$ MMed, PhD; S B P Mackenzie, ${ }^{4,5}$ MB ChB, FRCOG (UK) \\ ${ }^{1}$ Department of Health Sciences, Faculty of Health and Applied Sciences, Namibia University of Science and Technology, Windhoek, Namibia \\ ${ }^{2}$ Division of Medical Virology, Department of Pathology, Faculty of Medicine and Health Sciences, Stellenbosch University, Cape Town, South \\ Africa \\ ${ }^{3}$ National Health Laboratory Service, Tygerberg Hospital, Cape Town, South Africa \\ ${ }^{4}$ Department of Obstetrics and Gynaecology, School of Medicine, University of Namibia, Windhoek, Namibia \\ ${ }^{5}$ Department Obstetrics and Gynaecology, Ministry of Health and Social Services, Windhoek, Namibia
}

\section{Corresponding author: B E van der Colf (evandercolf@nust.na)}

Background. Seroprevalence of cytomegalovirus (CMV) is high in developing countries. However, a pregnant woman's immunity does not necessarily protect her baby against congenital CMV infection.

Objectives. To determine the seroprevalence of CMV among pregnant women attending a public antenatal clinic (Windhoek Central Hospital, Namibia) and subsequently determine the risk of vertical transmission and congenital CMV infection.

Methods. Blood samples and demographic information were collected from 344 pregnant women (age range 15 - 48 years). Serum was tested for anti-CMV IgG and IgM using an automated chemiluminescence assay, and an ELISA was used to assess specific IgG avidity. Fisher's exact test was used to determine associations among variables.

Results. Seroprevalence of anti-CMV IgG was found to be $100 \%$ across the study population, with positive or grey-zone anti-CMV IgM results found in 11 women (3.2\%). Specific IgG avidity was high in all cases. Neither maternal nor gestational age was positively associated with a positive or grey-zone IgM result. Parity was significantly associated with CMV IgM seroprevalence, with the highest level observed in women who had had one previous pregnancy.

Conclusion. This was the first study to investigate seroprevalence of CMV in Namibia. Despite the high seroprevalence among pregnant women, the burden of congenital CMV infection may be carried by infants in the Namibian population. This may contribute to long-term disabilities, especially sensorineural hearing loss. Further studies are needed to determine the prevalence of congenital CMV in Namibia.

S Afr J Obstet Gynaecol 2019;25(2):52-55. https://doi.org/10.7196/SAJOG.2019.v25i2.1441

Cytomegalovirus (CMV) infection in the first trimester of pregnancy is known to cause congenital malformation, especially of the central nervous system. CMV has been identified as the main cause of non-inherited hearing loss while long-term CNS sequelae include seizures and motor and visual defects. ${ }^{[1-3]}$ Between $10 \%$ and $20 \%$ of all children with congenital CMV (cCMV) infections may show signs of neurological damage during follow-up, ${ }^{[3,4]}$ and Enders et al. ${ }^{[5]}$ reported that $57.6 \%$ of congenitally infected live-born infants had symptoms of varying degree. Congenitally infected newborns are at risk of serious long-term sequelae regardless of the presence or absence of symptoms at birth. ${ }^{[2,6]}$ Transmission of CMV infection occurs through breastfeeding, sexual contact and contact with body fluids. ${ }^{[7]}$ cCMV infection may develop when a pregnant woman is infected with CMV. Primary or non-primary maternal infection (reactivation or reinfection) at any time during pregnancy can lead to CMV crossing the placenta and infecting the fetus. ${ }^{[8]}$

Owing to variations in epidemiology and seropositivity in women of childbearing age, prevalence of cCMV infection varies across countries. In the USA, epidemiologic studies suggest that CMV infection occurs in approximately $1 \%$ of all births or affects about 40000 infants annually. These CMV infections are asymptomatic in most cases. ${ }^{[9]}$ Prevalence of cCMV infection was found to be
4.6/1 000 births in Sweden and 3.2/1 000 births in London. ${ }^{[10]}$ Bonalumi et al. ${ }^{[3]}$ reported an incidence of $0.3-2.4 \%$ in developed countries. The incidence of cCMV infection is highest in developing countries ( 1 - 5\% of births) and can most likely be attributed to nonprimary maternal infections..$^{[4,11,12]}$

CMV infection in pregnant women can be either primary or recurrent. Primary infection occurs when a person is infected with the virus for the first time and can be demonstrated by seroconversion (appearance of antibodies that were not previously present). Recurrent infection develops when a previous, latent infection reactivates or if reinfection with a different CMV strain occurs. During latency there is no viral replication, although viral products may be transcribed during this time. The virus remains dormant in mononuclear leukocytes and cells of organs such as the kidneys and heart. The virus may start replicating at any time, causing a reactivation of the infection. ${ }^{[2]}$

Recent data demonstrate a similar risk of sequelae, especially hearing loss, developing in infants born to mothers with primary CMV infection or CMV reinfection. There is increasing evidence that non-primary maternal infection could lead to symptomatic and severe outcomes. This underscores the importance of screening for cCMV infection in resource-limited settings. In well-resourced 
settings, it is believed that primary maternal infection drives cCMV infection; however in resource-limited settings with a high prevalence and incidence, reinfections may contribute to congenital infections. ${ }^{[3,13]}$

\section{Methods}

A total of 344 pregnant women attending public antenatal care in Windhoek, Namibia, during 2016 were enrolled in the study. Serum was tested for CMV IgG and IgM by means of an automated chemiluminescence assay (Access Immunoassay Systems, Beckman Coulter, Switzerland). IgG avidity was assessed by ELISA (Euroimmun, Germany). The reference range for negative CMV IgG was $<6.0 \mathrm{AU} / \mathrm{mL}$. For specific IgM, a grey-zone value meant that the value was not negative but also not high enough to confidently be classified as positive. Software for statistical analysis included SPSS (version 24) and $\mathrm{R}$ (version 3.2.2). ${ }^{[14]}$ Fisher's exact test was used to determine $p$-values. A significance level of $p<0.05$ was used.

\section{Ethical approval}

Ethical approval was granted by the School of Health and Applied Sciences at the Namibia University of Science and Technology, the Namibian Ministry of Health and Social Services (ref. no. 17/3/3) and by the Health Research Ethics Committee of Stellenbosch University (ref. no. S16/05/092; IRB0005239).

\section{Results}

The seroprevalence of anti-CMV IgG was $100 \%$ across the study population. Results from 11 women (3.2\%) were positive (or in the grey zone) for IgM (Table 1). Neither maternal nor gestational age was associated with IgM activation. A significant association was found between parity and CMV IgM seroprevalence, with the highest level in women who had had one previous pregnancy. This could possibly be attributed to a higher probability of risky behaviour and higher exposure to CMV in younger age groups.

Evidence of longstanding CMV infection was found in all samples. All IgM-positive samples $(n=11)$ showed high-avidity IgG antibodies against CMV, indicating previous infections (Table 2).

\section{Discussion}

Seroprevalence of CMV infection in our study was higher than what is generally reported for Europe and North America ( 50\%), but similar to prevalences seen in South America and elsewhere in Africa. ${ }^{[2,7,15-18]}$ In high-prevalence settings, most primary CMV infections occur in infancy. In a recent South African study, polymerase chain reaction (PCR) analysis of saliva samples showed $2.9 \%$ of newborns from HIV-positive mothers to be CMV infected ${ }^{[11]}$ in another, cCMV infection was found in $5.96 \%$ of newborns, but without a significant association with the mother's HIV status. ${ }^{[19]}$ A population-based study of 460 healthy infants in Zambia showed $83 \%$ CMV seroprevalence by 18 months of age. ${ }^{[20,21]}$ Early infection (i.e. before reaching childbearing potential) could therefore explain the $100 \%$ prevalence in our study across age strata.

Despite all pregnant women in our analysis shown to have pre-existing anti-CMV antibodies, they remain at risk of CMV reactivation or reinfection during pregnancy and subsequent vertical transmission to the fetus. High maternal seroprevalence of CMV therefore does not exclude the threat of cCMV infection. Although individual risk is highest with primary infections, Ornoy and Diav-
Table 1. Anti-cytomegalovirus IgM positivity in relation to sociodemographic and antenatal characteristics among pregnant women attending a public antenatal clinic in Windhoek, Namibia, 2016 ( $N=344)$

\begin{tabular}{lll}
\hline Characteristics & $\begin{array}{l}\text { IgM-positive } \\
\text { samples, } \boldsymbol{n} / \mathbf{N}(\%)^{*}\end{array}$ & $\boldsymbol{p}$-value \\
\hline All participants & $11 / 344(3.2)$ & \\
Maternal age (years) & & \\
$15-20$ & $1 / 38(2.6)$ & 0.613 \\
$21-25$ & $5 / 108(4.6)$ & \\
$26-30$ & $2 / 96(2.1)$ & \\
$31-35$ & $3 / 59(5.1)$ & \\
36 - 48 & $0 / 43(0.0)$ & \\
Gestational age & & \\
First trimester & $1 / 41(2.4)$ & \\
Second trimester & $6 / 172(3.5)$ & \\
Third trimester & $4 / 130(3.1)$ & \\
Parity & & \\
Primigravida & $5 / 129(3.9)$ & \\
1 previous pregnancy & $6 / 100(6.0)$ & \\
$>2$ previous pregnancies & $0 / 115(0.0)$ & \\
&
\end{tabular}

Table 2. Anti-cytomegalovirus IgG and IgM seropositivity and IgG avidity among pregnant women

\begin{tabular}{llll}
\hline Case & $\begin{array}{l}\text { IgG } \\
\text { concentration } \\
(\text { AU/mL) }\end{array}$ & IgM status & IgG avidity \\
\hline 5011 & 241.2 & Grey zone & High \\
5014 & 140.0 & Grey zone & High \\
5020 & 46.0 & Positive & High \\
5049 & $>250.0$ & Grey zone & High \\
5127 & 181.7 & Positive & High \\
5133 & 205.3 & Grey zone & High \\
5165 & $>250.0$ & Positive & High \\
5188 & 248.3 & Grey zone & High \\
5287 & $>250.0$ & Positive & High \\
5295 & 213.5 & Positive & High \\
5348 & 114.0 & Grey zone & High \\
& & &
\end{tabular}

Citrin ${ }^{[22]}$ concluded that cCMV infection occurs in infants born to previously infected mothers. More than $60 \%$ of infants infected with CMV in utero appear to be born to mothers who were seropositive before conception and who experienced reactivation of latent virus or reinfection with a new strain during pregnancy. An increasing number of studies show severe sequelae in these infants. ${ }^{[8]}$ Maternal IgG positivity alone does not eliminate the risk of cCMV infection. It is thought that the cytotoxic T-lymphocyte response - another arm of the adaptive immune response - is more important than antibodies in preventing CMV reactivation and therefore treatment or conditions that result in a decreased cellular immunity are associated with CMV reactivation even in the presence of high IgG titres.

Leruez-Ville and Ville ${ }^{[23]}$ reported that the burden of cCMV infection can be attributed to secondary maternal infection to a larger extent than previously thought. Unfortunately, there are still no validated tools to accurately diagnose and differentiate maternal reinfection and reactivation. ${ }^{[23]}$ 
CMV IgM is a sensitive marker of primary CMV infection, but can also be detected during recurrent infection. False positives may develop owing to polyclonal activation or the presence of crossreactive antigens in the case of a primary Epstein-Barr infection. ${ }^{[2]}$ In the case of a primary CMV infection, the individual risk of transmission is highest, but in high-prevalence settings most cases of true CMVIgM positivity are due to secondary infections, when the individual transmission risk is lower. Taken together, detecting maternal CMV IgM does not mean that the fetus will be infected, and further testing is warranted in an attempt to differentiate a primary or recurrent infection or to identify another cause of CMV-IgM reactivity (e.g. primary Epstein-Barr infection)..$^{[2,25]}$

IgG and IgM serology of a pregnant woman can identify pregnancies at risk of transmitting CMV to the fetus. Finding CMV IgM antibodies with low IgG avidity supports a primary infection and a high risk of transmission, whereas in cases with high avidity, the individual risk of transmission is lower. ${ }^{[26]}$ When primary maternal infection has been diagnosed or when highdefinition ultrasound and magnetic resonance imaging suggest congenital infection, CMV DNA can be detected by means of PCR using amniotic fluid taken at 21 weeks of gestation. ${ }^{[3]}$ Neonatal diagnosis of cCMV infection is by viral culture or PCR on blood or urine within 2 weeks of birth. Cases with asymptomatic cCMV infection may require more frequent follow-up to diagnose sensorineural hearing loss early and to prevent further deterioration. ${ }^{[12]}$

However, the cost of comprehensive diagnosis and treatment of CMV infection is prohibitive in sub-Saharan Africa and there is a need for more affordable solutions. Moreover, there is limited evidence of the benefit of antenatal antiviral therapy which, owing to its toxicity, is seldom considered during pregnancy. In neonates, there is uncertainty about the indications for and duration of therapy. ${ }^{[27]}$ Studies on newer, less toxic drugs are ongoing. ${ }^{[28]} \mathrm{CMV}$ reactivation is common in immunocompromised hosts. Therefore, in settings with a high prevalence of HIV infection, the risk of reactivation is increased. ${ }^{[29]}$ Despite these challenges, clinical trials with vaccines, prenatal interventions and prolonged postnatal antiviral therapy are underway. This emphasises the need for more information on the epidemiology and diagnosis of CMV infections in pregnant women and neonates. ${ }^{[13]}$

\section{Conclusion}

All pregnant women in our study presented with IgG antibodies for CMV. Of the women, 11 showed IgM antibodies for CMV, which could have been a result of reactivation of the infection or reinfection with a new strain of CMV. IgG avidity was high in all cases, suggesting absence of primary infections. However, preexisting immunity does not exclude vertical transmission of the virus during pregnancy. Despite the high seroprevalence of CMV among pregnant women, the Namibian population might carry the burden of cCMV infection among infants. This may contribute to long-term disability, especially sensorineural hearing loss. Further studies are needed to determine the prevalence of cCMV in Namibia and neonatal surveillance studies may be important to establish the prevalence of cCMV disease. Such information could lead to health interventions aimed at reducing disabilities such as hearing loss. Furthermore, emphasis should be on factors to be considered in designing further studies, for example the timing of viral infection.
Acknowledgements. Phlebotomists S Makura and E Nkhata are acknowledged for collecting samples and administering questionnaires. The authors also thank Pathcare Laboratories for sample analysis and the staff and participants at the antenatal clinic for their co-operation.

Author contributions. EvdC was responsible for study conceptualisation and design, data collection, analysis and interpretation, and drafting the manuscript. GvZ supervised the project and was responsible for critical revision of the manuscript. SM assisted with sample collection and reviewed the manuscript.

Funding. This work was supported by the National Commission on Research, Science and Technology in Namibia. The sponsors had no role in the study design, collection, analysis and interpretation of data, writing of the report or the decision to submit the article for publication.

Conflicts of interest. None.

1. Cheeran MC, Lokensgard JR, Schleiss MR. Neuropathogenesis of congenital cytomegalovirus infection: Disease mechanisms and prospects for intervention. Clin Microbiol Rev 2009;22(1):99126. https://doi.org/10.1128/cmr.00023-08

2. Ijpelaar H. Prenatal diagnosis of cytomegalovirus infection. http://www.siemens.com/diagnostics (accessed 3 June 2013).

3. Bonalumi S, Trapanese A, Santamaria A, D’Emidio L, Mobili L. Cytomegalovirus infection in pregnancy: Review of the literature. J Prenat Med 2011;5(1):1-8.

4. Malm G, Engman M-L. Congenital cytomegalovirus infections. Semin Fetal Neonatal Med 2007;12(3):154-159. https://doi.org/10.1016/j.siny.2007.01.012

5. Enders G, Bader U, Lindemann L, Schalasta G, Daiminger A. Prenatal diagnosis of congenital cytomegalovirus infection in 189 pregnancies with known outcome. Prenat Diag 2001;21(5):362377. https://doi.org/10.1002/pd.59

6. Kenneson A, Cannon MJ. Review and meta-analysis of the epidemiology of congenital cytomegalovirus (CMV) infection. Rev Med Virol 2007;17(4):253-276. https://doi.org/10.1002/rmv.535

7. Al-Jiffri O, Al-Sharif FM, El-Sayed ZM. Seroprevalence of cytomegalovirus among blood donors and other investigated groups. Int J Microbiol Res 2013;4(1):01-08.

8. Wang C, Zhang X, Bialek S, Cannon MJ. Attribution of congenital cytomegalovirus infection to primary versus non-primary maternal infection. Clin Infect Dis 2011;52:e11-e13. https://doi. org/10.1093/cid/ciq085

9. Nelson K. Epidemiology of infectious disease: General principles. In: Nelson K, Williams CM, eds. Infectious Disease Epidemiology: Theory and Practice. 2nd ed. Burlington: Jones and Bartlett, 2007:25-62.

10. Townsend CL, Forsgren M, Ahlfors K, Ivarsson S-A, Tookey PA, Peckham CS. Long-term outcomes of congenital cytomegalovirus infection in Sweden and the United Kingdom. Clin Infect Dis 2013;56(9):1232-1239. https://doi.org/10.1093/cid/cit018

11. Manicklal S, Van Niekerk AM, Kroon SM, et al. Birth prevalence of congenital CMV among infants of HIV-infected women on prenatal antiretroviral prophylaxis in South Africa. Clin Infect Dis 2014;58(10):1467-1472. https://doi.org/10.1093/cid/ciu096

12. Goderis J, De Leenheer E, Smets K, Van Hoecke H, Keymeulen A, Dhooge I. Hearing loss and congenital CMV infection: A systematic review. Pediatrics 2014;134(5):972-982.

13. Manicklal S, Emery VC, Lazzarotto T, Boppana SB, Gupta RK. The 'silent' global burden of congenital cytomegalovirus. Clin Microbiol Rev 2013;26(1):86-102. https://doi.org/10.1128/ CMR.00062-12. https://doi.org/10.1542/peds.2014-1173

14. R Development Core Team. R: A language and environment for statistical computing. Vienna: R Foundation for Statistical Computing, 2008. http://www.R-project.org.

15. Rodier MH, Berthonneau J, Bourgoin A, et al. Seroprevalences of toxoplasma, malaria, rubella, cytomegalovirus, HIV and treponemal infections among pregnant women in Cotonou, Republic of Benin. Acta Tropica 1995;59(4):271-277. https://doi.org/10.1016/0001-706x(95)00087-u

16. Adjei AA, Armah HB, Gbagbo F, Boamah I, Adu-Gyamfi C, Asare I. Seroprevalence of HHV-8, $\mathrm{CMV}$, and EBV among the general population of Ghana, West Africa. BMC Infect Dis 2008;8:111. https://doi.org/10.1186/1471-2334-8-111

17. Akinbami AA, Akanmu AS, Adeyemo TA, Wright KO, Dada MO, Dosunmu AO. Cytomegalovirus antibodies amongst healthy blood donors at Lagos University Teaching Hospital. S Afr Med J 2009;99(7):528-530.

18. Kafi SK, Eldouma EY, Saeed SBM, Musa HA. Seroprevalence of cytomegalovirus among blood donors and antenatal women attending two hospitals in Khartoum State. Sudan J Med Sc 2009;4(4):399-401. https://doi.org/10.4314/sjms.v4i4.51005

19. Tshabalala D, Newman H, Businge C, Mabunda SA, Kemp W, Beja P. Prevalence and determinants of congenital cytomegalovirus infection at a rural South African central hospital in the Eastern Cape. S Afr J Infect Dis 2018;33(4):89-92. https://doi.org/10.4102/sajid.v33i4.155

20. Bates M, Brantsaeter AB. Human cytomegalovirus (CMV) in Africa: A neglected but important pathogen. J Virus Erad 2016;2(3):136-142.

21. Gompels UA, Larke N, Sanz-Ramos M, et al. Human cytomegalovirus infant infection adversely affects growth and development in maternally HIV-exposed and unexposed infants in Zambia. Clin Infect Dis 2012;54(3):434-442. https://doi.org/10.1093/cid/cir837

22. Ornoy A, Diav-Citrin O. Fetal effects of primary and secondary cytomegalovirus infection in pregnancy. Reprod Toxicol 2006;21(4):399-409. https://doi.org/10.1016/j.reprotox.2005.02.002

23. Leruez-Ville M, Ville Y. Fetal cytomegalovirus infection. Best Pract Res Clin Obstet Gynaecol 2017;38:97-107. https://doi.org/10.1016/j.bpobgyn.2016.10.005

24. Lang D, Vornhagen R, Rothe M, Hinderer W, Sonneborn H-H, Plachter B. Cross-reactivity of Epstein-Barr virus-specific immunoglobulin $M$ antibodies with cytomegalovirus antigen containing glycine homopolymers. Clin Diagn Lab Immunol 2001;8(4):747-756. https://doi. org/10.1128/CDLI.8.4.747-756.2001

25. Yamamoto AY, Mussi-Pinhata MM, Boppana SB, et al. Human cytomegalovirus reinfection is associated with intrauterine transmission in a highly cytomegalovirus-immune maternal population. Am J Obstet Gynecol 2010;202(3):297.e1-8. https://doi.org/10.1016/j.ajog.2009.11.018

26. Blackburn NK, Besselaar TG, Schoub BD, O'Connell KF. Differentiation of primary cytomegalovirus infection from reactivation using the urea denaturation test for measuring antibody avidity. J Med Virol 1991;33(1):6-9. https://doi.org/10.1002/jmv.1890330103 


\section{RESEARCH}

27. Hamilton ST, Van Zuylen W, Shand A, et al. Prevention of congenital cytomegalovirus complications https:/ldoiorg/101002/rmveat

28. Morère L, Andouard D, Labrousse F, et al. Ex vivo model of congenital cytomegalovirus Morère L, Andouard D, Labrousse F, et al. Ex vivo model of congenital cytomegalovirus
infection and new combination therapies. Placenta 2015;36(1):41-47. https://doi.org/10.1016/j.
29. Laher F, Ashford G, Cescon A, et al. Held to ransom - CMV treatment in South Africa. S Afr J HIV Med 2010;11(1):31-34. https://doi.org/10.4102/sajhivmed.v1li1.243

Accepted 25 August 2019 